Document downloaded from:

http://hdl.handle.net/10251/139235

This paper must be cited as:

Blanes Zamora, S. (12-2). On the construction of symmetric second order methods for ODEs. Applied Mathematics Letters. 98:41-48. https://doi.org/10.1016/j.aml.2019.05.026

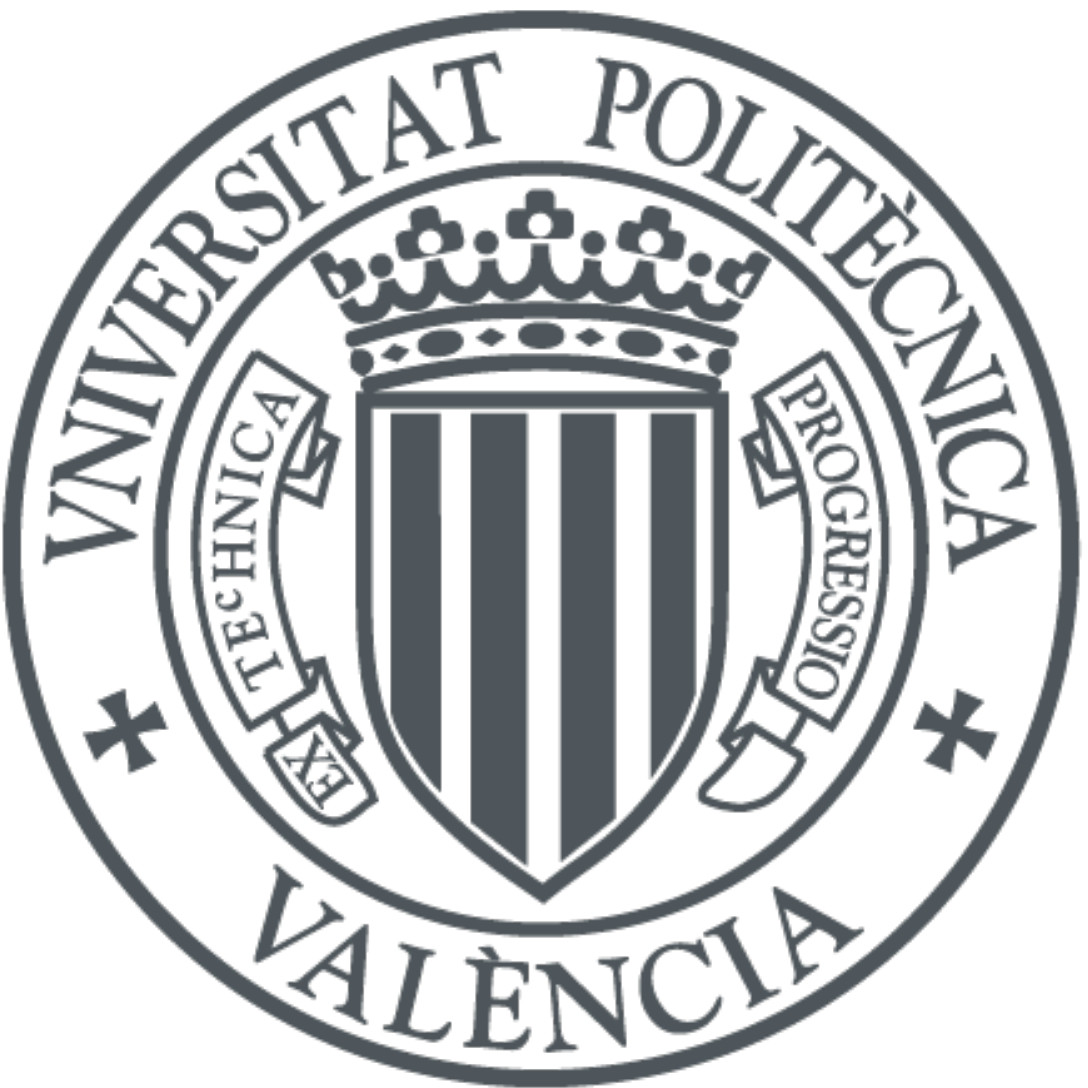

The final publication is available at

https://doi.org/10.1016/j.aml.2019.05.026

Copyright Elsevier

Additional Information 


\title{
On the construction of symmetric second order methods for ODEs
}

\author{
Sergio Blanes \\ Instituto Universitario de Matemática Multidisciplinar, Universitat Politècnica de València. Spain
}

\begin{abstract}
We show how to build explicit symmetric second order methods for solving ordinary differential equations. These methods are very useful when low accuracy is required or when higher order ones by extrapolation or composition are desired to reach high accuracy. The proposed schemes are obtained by using simple splitting methods on an extended phase space. By construction, the schemes are symmetric and of second order allowing to recover most well known and frequently used schemes from the literature. This provides a simple proof on their time symmetric structure that is very useful when the schemes are used to get higher order methods by extrapolation or composition. We show how to obtain them in the general case as well as how to get Nyström methods, methods for stiff problems, Lie group integrators or symplectic integrators, but the technique can also be used to build explicit and implicit methods for many other problems.
\end{abstract}

Keywords: Numerical methods for ODEs; symmetric second order methods; extrapolation; composition methods

\section{Introduction}

In this letter we consider the numerical integration of the ODE

$$
x^{\prime}=f(t, x), \quad x\left(t_{0}\right)=x_{0},
$$

with $x \in \mathbb{C}^{d}$ and formal solution given by $x(t)=\phi_{t}\left(x_{0}\right)$. In general, standard Runge-Kutta, multistep and extrapolation methods are the numerical schemes chosen to solve this problem [6, 12, 13, 14, 23]. However, when the ODE has some particular structure, tailored methods are considered. For example:

- If (1) originates from the second order ODE

$$
y^{\prime \prime}=g(t, y), \quad y\left(t_{0}\right)=y_{0}, y^{\prime}\left(t_{0}\right)=y_{0}^{\prime},
$$

Nyström methods are frequently used. If (1) is written as

$$
\frac{d}{d t}\left(\begin{array}{c}
y \\
y^{\prime}
\end{array}\right)=\left(\begin{array}{c}
y^{\prime} \\
g(t, y)
\end{array}\right)
$$

RK, multistep or extrapolation methods can be optimized to solve this problem [12].

- If the problem (1) is stiff, then appropriate implicit methods are usually required $[6,13,14]$.

- If $f(t, x)$ has a given geometric structure such that the solution has some qualitative properties, i.e. the map $\phi_{t}$ is a unitary, orthogonal, symplectic, ... flow then Geometric Integrators are the best choice, i.e. methods that preserve some of the qualitative properties of the exact flow $[1,11,15,16,18,20]$.

Email address: serblaza@imm. upv.es (Sergio Blanes) 
When low accuracy is desired, first or second order methods in the time step suffice since typically they allow to use relatively long time steps at a low computational cost. Among them, symmetric second order methods (when available) are preferred because, in general, provide the best rate between accuracy and computational cost. In many cases they have also shown better error propagation (this is the case for the well known Strang, Störmer, Verlett, leapfrog, midpoint, trapezoidal, Crank-Nicolson, etc., methods) but, obviously, the most appropriate method for solving a given equation depends on each particular problem.

On the other hand, when accurate results are desired, high order methods usually provide the best performance. Among them, extrapolation and composition methods have shown to be highly competitive. These schemes are based on a low order method and, either by appropriate linear combination of the methods used with different time steps with extrapolation [12] or by composition of them [1,11, 17, 25] it is possible to reach higher order schemes. The best performance is usually reached when the low order scheme is a symmetric second order method. Obviously, one has to use a basic method that is as accurate, cheap and stable as possible.

There are in the literature many different symmetric second schemes built for solving a wide class of problems. To construct second order methods and to prove their time symmetry has been quite cumbersome in some cases. We present a unified and simplified procedure that allows us to recover most of the schemes from the literature (built in many cases for autonomous problems), and we extend these schemes to non-autonomous problems as well as we build new ones.

\section{Symmetric second order methods}

We first review some of the well known symmetric second order methods from the literature that are also frequently used to obtain higher order ones. Let $\psi_{h}$ the numerical flow defined by a method to advance a time step $h$, i.e. $x_{k+1}=\psi_{h}\left(x_{k}\right)$ with $x_{k} \simeq x\left(t_{k}\right), t_{k}=t_{0}+k h$. If $\psi_{h}^{-1}=\psi_{-h}$ the method is selfadjoint, i.e. $x_{k}=\psi_{-h}\left(x_{k+1}\right)$, the error can be expanded in terms of even powers of $h$, and we say the method is time-symmetric.

- Gragg proved in [10] that the quantity $S_{h}(x)$ produced by the algorithm $\left(t=t_{0}+2 n h, t_{i}=t_{0}+i h\right)$

$$
\begin{aligned}
x_{1} & =x_{0}+h f\left(t_{0}, x_{0}\right) \\
x_{i+1} & =x_{i-1}+h f\left(t_{i}, x_{i}\right), \quad i=1,2, \ldots, 2 n \\
S_{h} & =\frac{1}{4}\left(x_{2 n-1}+2 x_{2 n}+x_{2 n+1}\right),
\end{aligned}
$$

is consistent and possesses an asymptotic expansion in even powers of $h$. This led to the construction of the Gragg-Bulirsch-Stoer-extrapolation algorithm (GBS) [12]. If a method of order $2 k$ is desired following the sequence $n=n_{1}, n_{2}, \ldots, n_{k}$, the number of evaluations of the vector field, $f$ is (notice that $f\left(t_{0}, x_{0}\right)$ needs to be computed only once) $c_{f}=1+2\left(n_{1}+n_{2}+\cdots+n_{k}\right)$. Gragg's proof of this property was very long and complicated, but Stetter [21] had the elegant idea of interpreting (1) as a one-step algorithm by rewriting (4) in terms of odd and even indices: for this purpose, if one defines $h^{*}=2 h, t_{k}^{*}=t_{0}+k h^{*}, u_{0}=v_{0}=y_{0}, u_{k}=$ $x_{2 k}, v_{k}=\frac{1}{2}\left(x_{2 k-1}+x_{2 k+1}\right)$, the method (4) can be rewritten as

$$
\left(\begin{array}{c}
u_{k+1} \\
v_{k+1}
\end{array}\right)=\left(\begin{array}{c}
u_{k} \\
v_{k}
\end{array}\right)+h^{*}\left(\begin{array}{c}
f\left(t_{k}^{*}+\frac{h^{*}}{2}, v_{k}+\frac{h^{*}}{2} f\left(t_{k}^{*}, u_{k}\right)\right) \\
\frac{1}{2}\left(f\left(t_{k}^{*}+h^{*}, u_{k+1}\right)+f\left(t_{k}^{*}, u_{k}\right)\right)
\end{array}\right),
$$

where the symmetry can be checked analytically by exchanging $u_{k+1} \leftrightarrow u_{k}, v_{k+1} \leftrightarrow v_{k}, h^{*} \leftrightarrow-h^{*}, t_{k}^{*} \leftrightarrow$ $t_{k}^{*}+h^{*}$. Then, the known as the smoothing step (that has better stability properties) is given by

$$
S_{h}\left(t_{0}+2 n h\right)=\frac{1}{4}\left(x_{2 n-1}+2 x_{2 n}+x_{2 n+1}\right)=\frac{1}{2}\left(u_{k}+v_{k}\right)
$$

- The Gragg's method can be used to solve (2) if written as (3). One can halve the number of evaluations of the 
function $g$ if one adapts the scheme as follows [12]

$$
\begin{aligned}
y_{1} & =y_{0}+h y_{0}^{\prime} \\
y_{1}^{\prime} & =y_{0}^{\prime}+h g\left(t_{0}, y_{0}\right) \\
y_{i+1} & =y_{i-1}+2 h y_{i-1}^{\prime}, \quad i=1,3, \ldots, 2 n-1 \\
y_{i+1}^{\prime} & =y_{i-1}^{\prime}+2 h g\left(t_{i}, y_{i}\right), \quad i=2,4, \ldots, 2 n \\
S_{h} & =2 y_{2 n} \\
S_{h}^{\prime} & =\frac{1}{2}\left(y_{2 n-1}^{\prime}+y_{2 n+1}^{\prime}\right),
\end{aligned}
$$

and eliminating the $y_{j}^{\prime}$-values one obtains the equivalent well known formula

$$
y_{i+2}-2 y_{i}+y_{i-2}=(2 h)^{2} g\left(t_{i}, y_{i}\right),
$$

Since only even indices appear, it is natural to write the method as follows

$$
y_{i+1}-2 y_{i}+y_{i-1}=h^{2} g\left(t_{i}, y_{i}\right),
$$

$i=1,2,3, \ldots, y_{i} \simeq y\left(t_{i}\right), t_{i}=t_{0}+i h$, that has to be initiated with $y_{1}=y_{0}+\frac{h}{2} g\left(t_{0}, y_{0}\right)$. This scheme is clearly time-symmetric and is often called the Störmer-Verlet method. This method has excellent qualitative properties and low error growth when applied to Hamiltonian systems. It is a symplectic integrator.

- Splitting methods. Suppose the vector field in (1) is autonomous and can be decomposed into a sum of two contributions, $f(x)=f^{[1]}(x)+f^{[2]}(x)$, in such a way that each sub-problem

$$
\dot{x}=f^{[i]}(x), \quad x(0)=x_{0} \in \mathbb{C}^{d}, \quad i=1,2
$$

can be integrated exactly (or, more generally, it is simpler to integrate than the original system), with solutions $x(h)=\varphi_{h}^{[i]}\left(x_{0}\right)$ at $t=t_{0}+h$. Then, a consistent and symmetric composition of these flows provides a symmetric second-order approximation to the exact solution [1, 18]. For example

$$
S_{h}=\varphi_{\frac{h}{2}}^{[1]} \circ \varphi_{h}^{[2]} \circ \varphi_{\frac{h}{2}}^{[1]}, \quad \text { i.e. } \quad x_{1}=\varphi_{\frac{h}{2}}^{[1]}\left(\varphi_{h}^{[2]}\left(\varphi_{\frac{h}{2}}^{[1]}\left(x_{0}\right)\right)\right)
$$

is a time symmetric second order method. If we define the Lie operators $\hat{A}^{[i]} \equiv f^{[i]}(x) \cdot \nabla, i=1,2$, then the exact solution is given by $\phi_{h}=\mathrm{e}^{h\left(\hat{A}^{[1]}+\hat{A}^{[2]}\right)}$ and the method can formally be written as

$$
S_{h}=\mathrm{e}^{\frac{h}{2} \hat{A}^{[1]}} \mathrm{e}^{h \hat{A}^{[2]}} \mathrm{e}^{\frac{h}{2} \hat{A}^{[1]}}=\mathrm{e}^{h\left(\hat{A}^{[1]}+\hat{A}^{[2]}+h^{2} \hat{E}_{2}+h^{4} E_{4}+\cdots\right)}
$$

( $\hat{E}_{k}$ are error terms that depend on $\hat{A}^{[i]}$ but not on $h$ ) that is obviously selfadjoint, the error can be expanded in even powers of $h$ and it is time-symmetric. In the non-autonomous case, $f(t, x)=f^{[1]}(t, x)+f^{[2]}(t, x)$, where $x(h)=\varphi_{h}^{[i]}\left(\tau, x_{0}\right)$ denotes the solution at $t=t_{0}+h$ of the autonomous equation, $\dot{x}=f^{[i]}(\tau, x)$, with $\tau$ a constant parameter (a frozen time), we can consider the time as two new dependent variables as follows

$$
\frac{d}{d t}\left(\begin{array}{c}
x \\
t_{1} \\
t_{2}
\end{array}\right)=\left(\begin{array}{c}
f^{[1]}\left(t_{1}, x\right) \\
0 \\
1
\end{array}\right)+\left(\begin{array}{c}
f^{[2]}\left(t_{2}, x\right) \\
1 \\
0
\end{array}\right)
$$

and the scheme can be written as the sequence

$$
\begin{aligned}
& z_{1}=\varphi_{\frac{h}{2}}^{[1]}\left(t_{0}, x_{0}\right) \\
& z_{2}=\varphi_{h}^{[2]}\left(t_{0}+\frac{h}{2}, z_{1}\right) \\
& x_{1}=\varphi_{\frac{h}{2}}^{[1]}\left(t_{0}+h, z_{2}\right) .
\end{aligned}
$$

Two consecutive steps of the method to obtain $x_{2}$ can be written as $S_{h} \circ S_{h}=\varphi_{\frac{h}{2}}^{[1]} \circ \varphi_{h}^{[2]} \circ \varphi_{h}^{[1]} \circ \varphi_{h}^{[2]} \circ \varphi_{\frac{h}{2}}^{[1]}$ so only one evaluation of $\varphi_{h}^{[1]}$ and $\varphi_{h}^{[2]}$ per step is required except for the first step. 
The splitting method, by construction, allows to easily build symmetric second order methods. It requires, however, that the vector field must be decomposed into solvable parts (or into parts that are easy to numerically approximate by methods that preserve the whole symmetry).

\section{Symmetric second order methods in a extended phase space}

In [4] it is shown the interest to consider the independent variable, $t$, in a non-autonomous vector field, $f(t, x)$, that is separable into solvable parts, as two or more different dependent variables. On the other hand, in [21] (see also [12]) it is shown that (4) is consistent with the differential equation

$$
\begin{array}{ll}
u^{\prime}=f(t, v), & u\left(t_{0}\right)=x_{0} \\
v^{\prime}=f(t, u), & v\left(t_{0}\right)=x_{0} .
\end{array}
$$

Notice that both equations share the same $t$ variable in the vector field. Then, the scheme (4) is recovered when these equations are solved with a particular symmetric second order method. To prove that such scheme is symmetric is not obvious being also difficult to generalize to other problems. In the conclusions of [21] the author also claims "It is quite obvious that the results of this paper are not of great practical importance. Since it is fully explicit Gragg's algorithm (4) is so ideally suited as a basis for RICHARDSON extrapolation that no other symmetric two-step algorithm can compete with it".

However, if we combine both procedures (to duplicate the system and to consider the time as two new dependent coordinates) we can lead to differential equations with the same solution as desired that are trivially solvable with splitting methods. This procedure allows to present a unified and simplified procedure to build methods. We can recover many of the existing methods as well as to build new symmetric schemes that can be easily adapted to solve different classes of problems, and this is illustrated on several problems.

\subsection{Symmetric second order methods for general problems}

Let us take in (11) $u_{t} \equiv t$ and $v_{t} \equiv t$ as two new coordinates as follows

$$
\frac{d}{d t}\left(\begin{array}{c}
u \\
u_{t} \\
v \\
v_{t}
\end{array}\right)=\left(\begin{array}{c}
0 \\
0 \\
f\left(u_{t}, u\right) \\
1
\end{array}\right)+\left(\begin{array}{c}
f\left(v_{t}, v\right) \\
1 \\
0 \\
0
\end{array}\right)=f^{[1]}\left(u_{t}, u\right)+f^{[2]}\left(v_{t}, v\right), \quad\left(\begin{array}{c}
u\left(t_{0}\right) \\
u_{t}\left(t_{0}\right) \\
v\left(t_{0}\right) \\
v_{t}\left(t_{0}\right)
\end{array}\right)=\left(\begin{array}{c}
x_{0} \\
t_{0} \\
x_{0} \\
t_{0}
\end{array}\right),
$$

whose solution is $u(t)=v(t)=x(t)$. This system is separable into two trivially solvable autonomous parts and splitting methods can be applied. We now show that the composition (10), corresponds to the Gragg's method (if one uses $n$ steps of the splitting method). Then, this provides a simple proof to show that the Gragg's method is consistent and symmetric, as desired: The scheme (10) applied to solve (12) is given by the sequence (from $t_{n}$ to $t_{n+1}$ with $u_{t, n}=v_{t, n}=t_{n} u_{n} \simeq u\left(t_{n}\right)$, etc. $)$

$$
\begin{aligned}
& \varphi_{\frac{h}{2}}^{[1]}: \begin{cases}v_{1 / 2}=v_{n}+\frac{h}{2} f\left(u_{t, n}, u_{n}\right) & \\
v_{t, 1 / 2}=v_{t, n}+\frac{h}{2} & v_{1 / 2}=v_{n}+\frac{h}{2} f\left(t_{n}, u_{n}\right)\end{cases} \\
& \varphi_{h}^{[2]}: \begin{cases}u_{n+1}=u_{n}+h f\left(v_{t, 1 / 2}, v_{1 / 2}\right) & u_{n+1}=u_{n}+h f\left(t_{n}+\frac{h}{2}, v_{1 / 2}\right) \\
u_{t, n+1}=u_{t, n}+h & v_{n+1}=v_{1 / 2}+\frac{h}{2} f\left(t_{n}+h, u_{n+1}\right),\end{cases} \\
& \varphi_{\frac{h}{2}}^{[1]}:\left\{\begin{array}{l}
v_{n+1}=v_{1 / 2}+\frac{h}{2} f\left(u_{t, n+1}, u_{n+1}\right) \\
v_{t, n+1}=v_{t, 1 / 2}+\frac{h}{2}
\end{array}\right.
\end{aligned}
$$

that agrees with (5) if we replace $t_{n}$ by $t_{k}^{*}$ and $h$ by $h^{*}$. If we concatenate $n$ steps of the splitting method we recover the Gragg's method. Obviously, both $u_{n}$ and $v_{n}$ are two different second order approximations to the exact solution, $x\left(t_{n}\right)$ so, its average, i.e. the smoothing, also corresponds to a symmetric second order approximation. This is a much simpler procedure to build methods and can be extended to solve different classes of problems as follows.

It is worth to notice that $u_{1}$ in the first step coincides with the explicit midpoint RK method. However, the second step to obtain $u_{2}$ using the midpoint RK method initiates with $u_{1}$ and do not take into account $v_{1}$, breaking the time symmetry of the method. In addition, the vector $\left(u_{n+1}, u_{t, n+1}, v_{n+1}, v_{t, n+1}\right)$ is obtained from $\left(u_{n}, u_{t, n}, v_{n}, v_{t, n}\right)$ 
trough a selfadjoint method but, if we take $u_{n}=v_{n}=x_{n}$ then the smoothing map $x_{n+1}=\frac{1}{2}\left(u_{n+1}+v_{n+1}\right)=$ $\psi_{h}\left(x_{n}\right)$ corresponds to a 3-stage explicit Runge-Kutta method. Then, the smoothing (although it can be used with extrapolation) makes that the method is not selfadjoint.

\subsection{Nyström symmetric second order methods}

Let us now consider the second order ODE (2) that we write as a first order ODE as in (3) but as follows

$$
\frac{d}{d t}\left(\begin{array}{c}
y \\
y_{t} \\
y^{\prime}
\end{array}\right)=\left(\begin{array}{c}
0 \\
0 \\
g\left(y_{t}, y\right)
\end{array}\right)+\left(\begin{array}{c}
y^{\prime} \\
1 \\
0
\end{array}\right),
$$

with $y_{t}\left(t_{n}\right)=t_{n}$. The splitting method (9) generates the scheme

$$
\begin{aligned}
& \varphi_{\frac{h}{2}}^{[1]}:\left\{\begin{array}{ll}
y_{1 / 2}^{\prime}=y_{n}^{\prime}+\frac{h}{2} g\left(y_{t, n}, y_{n}\right) & \\
\varphi_{h}^{[2]}: \begin{cases}y_{n+1}=y_{n}+h y_{1 / 2}^{\prime} & \text { or }\end{cases} \\
y_{t, n+1}=y_{t, n}+h & y_{n+1}=y_{n}+h y_{n}^{\prime}+\frac{h^{2}}{2} g\left(t_{n}, y_{n}\right)
\end{array} y_{n+1}^{\prime}=y_{n}^{\prime}+\frac{h}{2}\left(g\left(t_{n}, y_{n}\right)+g\left(t_{n}+h, y_{n+1}\right)\right)\right.
\end{aligned}
$$

that is equivalent to the symmetric second order method (6) or the Störmer method (7) that is used as the basic method for extrapolation (see [12]). The cost of the method, for a sequence of $n=n_{1}, n_{2}, \ldots, n_{k}$ to build a method of order $p=2 k$ by extrapolation is $c_{N}^{(1)}=1+n_{1}+n_{2}+\ldots+n_{k}$ evaluations of the function $g$ (since $g\left(t_{n}, y_{n}\right)$ can be stored and reused). On the other hand, since the maps $\varphi_{h}^{[1]}$ and $\varphi_{h}^{[2]}$ are qualitatively different, this simple procedure suggests to consider the following sequence $S_{h}=\varphi_{\frac{h}{2}}^{[2]} \circ \varphi_{h}^{[1]} \circ \varphi_{\frac{h}{2}}^{[2]}$ that corresponds to the scheme

$$
\begin{aligned}
& \varphi_{\frac{h}{2}}^{[2]}:\left\{\begin{array}{l}
y_{1 / 2}=y_{n}+\frac{h}{2} y_{n}^{\prime} \\
y_{t, 1 / 2}=y_{t, n}+\frac{h}{2}
\end{array}\right. \\
& \varphi_{h}^{[1]}:\left\{y_{n+1}^{\prime}=y_{n}^{\prime}+h g\left(y_{t, 1 / 2}, y_{1 / 2}\right)\right. \\
& \varphi_{\frac{h}{2}}^{[2]}:\left\{\begin{array}{l}
y_{n+1}=y_{1 / 2}+\frac{h}{2} y_{n+1}^{\prime} \\
y_{t, n+1}=y_{t, 1 / 2}+\frac{h}{2}
\end{array}\right. \\
& y_{n+1}^{\prime}=y_{n}^{\prime}+h g\left(t_{n}+\frac{h}{2}, y_{n}+\frac{h}{2} y_{n}^{\prime}\right) \\
& y_{n+1}=y_{n}+\frac{h}{2}\left(y_{n}^{\prime}+y_{n+1}^{\prime}\right)
\end{aligned}
$$

that has a slightly reduced cost of $c_{N}^{(2)}=c_{N}^{(1)}-1$ evaluations of $g$ when used with extrapolations and do not need to store the function $g$ that could be more efficient in some cases. Since in this case there is not smoothing, the schemes are selfadjoint. In spite the methods are not new, this procedure allows to build new schemes. For example, given the second order damped equation, $y^{\prime \prime}+\alpha(t) y^{\prime}=g(t, y)$ if we consider the split in the extended phase space

$$
\frac{d}{d t}\left(\begin{array}{c}
y \\
y_{1, t} \\
y^{\prime} \\
y_{2, t}
\end{array}\right)=\left(\begin{array}{c}
0 \\
0 \\
g\left(y_{1, t}, y\right) \\
1
\end{array}\right)+\left(\begin{array}{c}
y^{\prime} \\
1 \\
-\alpha\left(y_{2, t}\right) y^{\prime} \\
0
\end{array}\right)
$$

where both parts are exactly solvable, one can build a symmetric second order method that only requires one evaluation of $g$ per step. If one uses the Gragg's scheme two evaluations per step are required.

\subsection{Symmetric second order methods for stiff problems}

Many different procedures can be used for solving stiff problems depending on the stiffness of the problem and the computational cost of the vector field. In [13] a symmetric second order method is used that is consistent with the system

$$
\begin{array}{ll}
u^{\prime}=f(t, v)+J(u-v), & u\left(t_{0}\right)=x_{0} \\
v^{\prime}=f(t, u)+J(u-v), & v\left(t_{0}\right)=x_{0}
\end{array}
$$


with $J \simeq f^{\prime}\left(t_{n}, y_{n}\right)$, that is very similar to an splitting method as we will see. One can use many different variants, the most appropriate will obviously depend on the particular problem to be solved. For example, for midly stiff problems a simple scheme is obtained if we consider the system

$$
\frac{d}{d t}\left(\begin{array}{c}
u \\
u_{t} \\
v \\
v_{t}
\end{array}\right)=\left(\begin{array}{c}
f\left(v_{t}, v\right) \\
1 \\
0 \\
0
\end{array}\right)+\left(\begin{array}{c}
0 \\
0 \\
f\left(u_{t}, u\right)+J(u-v) \\
1
\end{array}\right)
$$

where the equation, $v^{\prime}=f\left(u_{t}, u\right)+J(u-v)$, with $u_{t}, u$ constant, can be solved either exactly

$$
v(t)=e^{t J} v(0)+h \varphi_{1}(h J)\left(f\left(u_{t}, u\right)+J u\right)
$$

where $\varphi_{1}(x)=\left(e^{x}-1\right) / x$, or one can use a symmetric implicit method, e.g.

$$
\left(I-\frac{h}{2} J\right) v_{n+1}=\left(I+\frac{h}{2} J\right) v_{n}+h\left(I-\frac{h}{2} J\right)\left(f\left(u_{t}, u\right)+J u\right) .
$$

Alternatively, since the vector field can be written as $f(t, x)=M(t, x) x$, with $M$ a matrix, and this can be done in many different ways, if there is a decomposition such that $M(t, x)$ is only mildly stiff, one can consider the system

$$
\begin{aligned}
& u^{\prime}=M\left(v_{t}, v\right) u, \\
& u_{t}^{\prime}=1, \\
& v^{\prime}=M\left(u_{t}, u\right) v, \\
& v_{t}^{\prime}=1,
\end{aligned}
$$

where the splitting methods leads to the scheme

$$
\begin{aligned}
u_{1 / 2} & =e^{\frac{h}{2} h M\left(t_{n}, v_{n}\right)} u_{n} \\
v_{n+1} & =e^{h M\left(t_{n}+\frac{h}{2}, u_{1 / 2}\right)} v_{n} \\
u_{n+1} & =e^{\frac{h}{2} M\left(t_{n+1}, v_{n+1}\right)} u_{1 / 2} .
\end{aligned}
$$

If we replace the exact maps by, for example, the midpoint rule (i.e. to approximate the exponentials by the second order diagonal Padé approximation) we get the scheme

$$
\begin{aligned}
\left(I-\frac{h}{4} M\left(t_{n}, v_{n}\right)\right) u_{1 / 2} & =\left(I+\frac{h}{4} M\left(t_{n}, v_{n}\right)\right) u_{n} \\
\left(I-\frac{h}{2} M\left(t_{n}+\frac{h}{2}, u_{1 / 2}\right)\right) v_{n+1} & =\left(I+\frac{h}{2} M\left(t_{n}+\frac{h}{2}, u_{1 / 2}\right)\right) v_{n} \\
\left(I-\frac{h}{4} M\left(t_{n}+h, v_{n+1}\right)\right) u_{n+1} & =\left(I-\frac{h}{4} M\left(t_{n}+h, v_{n+1}\right)\right) u_{1 / 2},
\end{aligned}
$$

where higher order methods can be obtained by composition or extrapolation.

\subsection{Symmetric second order Lie-group methods}

Suppose one knows that the solution of the ODE evolves on a differentiable manifold. For simplicity, let us consider the manifold is a Lie group of $n \times n$ matrices, and thus the vector field is of the form $f(t, x)=M(t, x) x$, with $M(t, x) \in g$ and $g$ is the linear subspace of the $n \times n$ matrices corresponding to the Lie algebra of the Lie group. Lie-group methods are frequently used to solve these problems [15]. We can solve the system (13) with the scheme (14) that can be considered as a second order commutator-free Lie group integrator [7, 8]. In the autonomous case, it corresponds to the second order method obtained in [24] used to build extrapolation methods in Lie groups. To preserve the Lie group structure one has either to compute very accurately the exponentials or to replace them by approximations that preserve this structure, like it is the case of diagonal Padés for most Lie groups. For instance, for unitary, orthogonal, symplectic, ... groups the scheme (14) is a symmetric second order Lie group integrator. Notice, however, that if one uses the average solution from $u_{n}, v_{n}$ as the smoothing method, the group structure breaks. 


\subsection{Symmetric second order symplectic methods}

Symplectic integrators have been successfully used to numerically solve Hamiltonian systems due to their qualitative properties and low error propagation $[1,11,16,20]$. For autonomous non-separable Hamiltonians, it has been recently proposed to solve another system in a extended phase space where the new Hamiltonian is separable into solvable parts $[19,22]$ while provide the desired solution. Given the non-autonomous Hamiltonian $H(t, q, p)$ with $q, p \in \mathbb{R}^{D}$ and the autonomous separable one

$$
K=\left(H\left(q_{t}^{(1)}, Q_{1},-Q_{2}\right)-q_{t}^{(2)}\right)+\left(H\left(p_{t}^{(2)}, P_{2}, P_{1}\right)+p_{t}^{(1)}\right)
$$

we have that $q_{t}^{(1)}=p_{t}^{(2)}=t, q(t)=Q_{1}(t)=P_{2}(t), p(t)=P_{1}(t)=-Q_{2}(t)$. In this case as well as in Lie group methods at each step we obtain two approximations to the solution. If the smoothing is considered the qualitative properties are destroyed because, in general, a linear combination of solutions do not preserve the group structure. This is also the case for extrapolation, although the qualitative properties are lost at higher orders than the order of the final method [2, 3]. In [22] this is solved by introducing into the Hamiltonian a new contribution to force that the numerical solutions for the pairs $Q_{1}(t), P_{2}(t)$ and $P_{1}(t),-Q_{2}(t)$ remain close to each other. For instance, to solve

$$
K=\left(H\left(q_{t}^{(1)}, Q_{1},-Q_{2}\right)-q_{t}^{(2)}\right)+\left(H\left(p_{t}^{(2)}, P_{2}, P_{1}\right)+p_{t}^{(1)}\right)+\frac{w}{2}\left(\left(Q_{1}-P_{2}\right)^{2}+\left(P_{1}+Q_{2}\right)^{2}\right)
$$

that is separable into three solvable parts (the last term corresponds to a linear harmonic oscillator) and the parameter $w$ has to be chosen appropriately for each problem. Non-separable Hamiltonians appear e.g. when considering variable time-step symplectic integrators using the Sundmann transformation (see [5] and references therein). Similar terms can also be introduced for solving the Lie group methods. Notice that the norm $\left\|u_{n+1}-v_{n+1}\right\|$ can also be used as an indicator of the error of the method.

\section{Acknowledgements}

This work has been funded by Ministerio de Economía, Industria y Competitividad (Spain) through project MTM2016-77660-P (AEI/FEDER, UE).

\section{References}

[1] S. Blanes and F. Casas, A Concise Introduction to Geometric Numerical Integration. CRC Press, Boca Raton, 2016.

[2] S. Blanes and F. Casas, Raising the order of geometric numerical integrators by composition and extrapolation, Numer. Alg., 38 (2005) 305-326.

[3] S. Blanes, F. Casas, and J. Ros, Extrapolation of symplectic integrators, Celest. Mech. Dyn. Astron., 75 (1999) $149-161$.

[4] S. Blanes, F. Diele, C. Marangi, and S. Ragni, Splitting and composition methods for explicit time dependence in separable dynamical systems. J. Comput. Appl. Math., 235 (2010) 646-659.

[5] S. Blanes and A. Iserles, Explicit Adaptive Symplectic Integrators for solving Hamiltonian Systems, Celest. Mech. Dyn. Astron., 114 (2012) 297-317.

[6] J.C. Butcher, Numerical Methods for Ordinary Differential Equations, Third revised ed., John Wiley \& Sons, Chichester, 2016.

[7] E. Celledoni, A. Marthinsen, and B. Owren, Commutator-free Lie group methods, Futur. Gener. Comput. Syst. 19 (2003) 341-352

[8] E. Celledoni, H. Marthinsen, and B. Owren, An introduction to Lie group integrators-basics, new developments and applications, J. Comput. Phys. 257 (part B) (2014) 1040-1061.

[9] P. Deuflhard, Recent progress in extrapolation methods for ordinary differential equations, SIAM Rev., 27 (1985) $505-535$.

[10] W.B. Gragg, On extrapolation algorithms for ordinary initial value problems, SIAM J. Num. Anal., ser.B, 2, (1965) $384-403$.

[11] E. Hairer, C. Lubich, and G. Wanner, Geometric Numerical Integration. Structure-Preserving Algorithms for Ordinary Differential Equations, 2nd edition, Springer-Verlag, 2006.

[12] E. Hairer, S.P. Nørsett, and G. Wanner, Solving Ordinary Differential Equations I. Nonstiff Problems, 2nd ed., Springer-Verlag, 1993.

[13] E. Hairer and G. Wanner, Solving Ordinary Differential Equations II. Stiff and Differential-Algebraic Problems, 2nd ed., Springer-Verlag, 1996.

[14] A. Iserles, A First Course in the Numerical Analysis of Differential Equations, 2nd ed. Cambridge University Press, Cambridge, 2008.

[15] A. Iserles, H. Z. Munthe-Kaas, S. P. Nørsett, and A. Zanna, Lie-group methods, Acta Numerica, 9, (2000) 215-365.

[16] B. Leimkuhler and S. Reich. Simulating Hamiltonian Dynamics. Cambridge University Press, 2004.

[17] R. I. McLachlan. On the numerical integration of ordinary differential equations by symmetric composition methods. SIAM J. Numer. Anal., 16 (1995) 151-168.

[18] R.I. McLachlan and R. Quispel. Splitting methods. Acta Numerica, 11 (2002) 341-434. 
[19] P. Pihajoki, Explicit methods in extended phase space for inseparable Hamiltonian problems, Celest. Mech. Dyn. Astron., 121 (2015) 211231.

[20] J. M. Sanz-Serna and M. P. Calvo. Numerical Hamiltonian Problems. AMMC 7. Chapman \& Hall, 1994.

[21] H.J. Stetter, Symmetric two-step algorithms for ordinary differential equations, Computing, 5 (1970) 267-280.

[22] M. Tao, Explicit symplectic approximation of nonseparable Hamiltonians: Algorithm and long time performance, Phys. Rev E, 94, 043303 (2016).

[23] J. Stoer and R. Bulirsch, Introduction to Numerical Analysis, 3rd ed. Springer Texts in Applied Mathematics 12, Springer-Verlag, 2002.

[24] J. Wensch, Extrapolation methods in Lie groups. Numer. Math. 89 (2001) 591-604.

[25] H. Yoshida. Construction of higher order symplectic integrators. Phys. Lett. A, 150 (1990) 262-268. 